\title{
Multifractal Analysis Revisited by the Continuous Wavelet Transform Applied in Lithofacies Segmentation from Well-Logs Data
}

\author{
Sid-Ali Ouadfeul and Leila Aliouane
}

\begin{abstract}
The main goal of this paper is to use the wavelet transform modulus maxima lines (WTMM) and the detrended fluctuations analysis (DFA) methods to establish a new technique of lithofacies segmentation from well logs data. The WTMM is used to delimitate lithoafacies boundaries and the DFA is used to provide an exact estimation of the roughness coefficient of lithofacies. Application of the proposed idea at the synthetic and real data of a borehole located in Berkine basin shows that the proposed technique can enhance reservoirs characterization.
\end{abstract}

Index Terms-WTMM, DFA, lithofacies, segmentation, reservoir.

\section{INTRODUCTION}

One of the main goals of geophysical studies is to apply suitable mathematical and statistical techniques to extract information about the subsurface properties. Well logs are largely used for characterizing reservoirs in sedimentary rocks. In fact it is one of the most important tools for hydrocarbon research for oil companies. Several parameters of the rocks can be analysed and interpreted in term of lithology, porosity, density, resistivity, salinity and the quantity and the kind of fluids within the pores.

Geophysical well-logs often show a complex behavior which seems to suggest a fractal nature [1],[2]. They are geometrical objects exhibiting an irregular structure at any scale. In fact, classifying lithofacies boundary from borehole data is a complex and non-linear problem. This is due to the fact that several factors, such as pore fluid, effective pressure, fluid saturation, pore shape, etc. affect the well log signals and thereby limit the applicability of linear mathematical techniques. To classify lithofacies units, it is, therefore, necessary to search for a suitable non-linear method, which could evade these problems.

The scale invariance of properties has led to the well known concept of fractals [3]. It is commonly observed that well $\log$ measurements exhibit scaling properties, and are usually described and modelled as fractional Brownian motions [1], [2], [4], [5], [6], [7], [8], [9], [10].

In previous works [11],[12], we have shown that well logs fluctuations in oil exploration display scaling behaviour

Manuscript received in May 14, 2011; revised June 25, 2011.

Sid-Ali Ouadfeul is with the Algeian Peroleum Institute, IAP Algeria and The Faculty of earth sciences, University of Sciences and technology, USTHB, Algeria(e-mail: Souadfeul@ymail.com).

Leila Aliouane is with University of M'hamed Bouguerra, Boumerdes, Algeria and The Faculty of earth sciences, University of Sciences and technology, USTHB, Algeria(email:1il_aldz@yahoo.fr). that has been modelled as self affine fractal processes. They are therefore considered as fractional Brownian motion $(\mathrm{fBm})$, characterized by a fractal $k^{-\beta}$ power spectrum model where $k$ is the wavenumber and $\beta$ is related to the Hurst exponent [13]. These processes are monofractal whose complexity is defined by a single global coefficient, the Hurst parameter $H$, which is closely related to the Hölder degree regularity Thus, characterizing scaling behaviour amounts to estimating some power law exponents.

Petrophysical properties and classification of lithofacies boundaries using the geophysical well log data is quite important for the oil exploration. Multivariate statistical methods such as principle component and cluster analyses and discriminant function analysis have regularly been used for the study of borehole data. These techniques are, however, semi-automated and require a large amount of data, which are costly and not easily available every time.

The wavelet transform modulus maxima lines is a type a multifractal analysis, where the mathematical measure is replaced by the modulus of the continuous wavelet transform(CWT), and the support of this measure is replaced by the points of maxima of the modulus of the CWT[14].

The detrended fluctuation analysis is a statistical method introduced by Peng[15] in genetic works, it was used for estimation of Hurst exponent of DNA nucleotides.

We use in this paper the WTMM combined with the DFA to establish a technique of lithofacies segmentation from well-logs data.

\section{PRINCIPAL OF THE DFA ESTIMATOR}

Method for quantifying the correlation propriety in no-stationary time series based on the computation of a scaling exponent $H$ by means of a modified root mean square analysis of a random walk[15].

To compute $H$ from a time-series $\mathrm{x}(i)[i=1, \ldots, \mathrm{N}]$, like the interval tachogram, the time series is first integrated:

$$
y(k)=\sum_{i=1}^{k}[x(i)-M]
$$

where $M$ is the average value of the series $\mathrm{x}(i)$, and $k$ ranges between 1 and $N$.

Next, the integrated series $\mathrm{y}(k)$ is divided into boxes of equal length $n$ and the least-square line fitting the data in each box, $\mathrm{y}_{\mathrm{n}}(k)$, is calculated. The integrated time series is detrended by subtracting the local trend $\mathrm{y}_{\mathrm{n}}(k)$, and the root-mean square fluctuation of the detrended series, $F(n)$ is computed:

$$
F(\mathrm{n})=\sqrt{\frac{1}{N} \sum_{k=1}^{N}\left[y(k)-y_{\mathrm{n}}(k)\right]^{2}}
$$


$\mathrm{F}(\mathrm{n})$ is computed for all time-scales $\mathrm{n}$.

Typically, $\mathrm{F}(\mathrm{n})$ increases with $\mathrm{n}$, the "box-size". If $\log \mathrm{F}(\mathrm{n})$ increases linearly with $\log \mathrm{n}$, then the slope of the line relating $\mathrm{F}(\mathrm{n})$ and $\mathrm{n}$ in a $\log -\log$ scale gives the scaling exponent $H$.

where : $\alpha=2 . H-1$

If $H=0.5$, the time-series $\mathrm{x}(i)$ is uncorrelated (white noise).

If $H=1.0$, the correlation of the time-series is the same of $1 / \mathrm{f}$ noise.

If $H=1.5, \mathrm{x}(i)$ behaves like Brown noise (random walk)

\section{COMPARISON BETWEEN DFA AND VARIOGRAMS}

A variogram is a geostatistical method of comparing similarity of a data value to neighbouring values within a field of data [16]. The variogram is calculated by:

$$
\gamma(h)=\frac{1}{N} * \sum_{i=1}^{N}\left[Z\left(x_{i}\right)-Z\left(x_{i+h}\right)\right]^{2}
$$

where $\mathrm{N}$ is the number of neighbouring data points within the specified lag distance being compared.

$\mathrm{Z}(\mathrm{x})$ : is the physical property parameter value of the initial point.

$\mathrm{Z}\left(\mathrm{x}_{\mathrm{i}+\mathrm{h}}\right)$ : is the parameter value of the neighbouring point.

The value $\gamma(h)$ is then plotted against versus the log distance between the initial point and the compared points. The resulting plot results in a curve where the variogram value increases with distance to a maximum, and levels off at a $\log$ distance where the total variability of the data field is reached. The geostatistical term for this distance is the range and is the correlation length discussed here.

A comparison between DFA and variograms at $\mathrm{fBm}$ realizations shows that:

-For signal of high number of samples (more than 1024) variograms gave better estimations of Hurst exponents compared to the DFA.

-For signal of low number of samples we distinguish two cases:

a) For signals of low Hurst exponent variograms method gives better results than the DFA.

b) For signals of High Hurst exponents, DFA estimate better the Hurst exponent

\section{THE WAVELET TRANSFORM MODULUS MAXIMA LINES}

The WTMM is composed of five steps [14]

a- Calculation of the continuous wavelet transform.

b- Calculation of the local maxima of the modulus of the CWT.

c- Calculation of the function of partition $\mathrm{Z}(\mathrm{q}, a)$ where $a$ is the dilatation and $\mathrm{q}$ is a scale factor.

d- Estimation of the spectrum of exponents $\tau(q)$.

e- Estimation of the spectrum of singularities $D(h)$.

\section{A. THE CONTINUOUS WAVELET TRANSFORM}

Here we review some of the important properties of wavelets, without any attempt at being complete. What makes this transform special is that the set of basis functions, known as wavelets, are chosen to be well-localized (have compact support) both in space and frequency [14],[17]. Thus, one has some kind of "dual-localization" of the wavelets. This contrasts the situation met for the Fourier Transform where one only has "mono-localization", meaning that localization in both position and frequency simultaneously is not possible.

The CWT of a function $\mathrm{s}(\mathrm{z})$ is given by Grossmann and Morlet [18] as:

$$
C_{s}(a, b)=\frac{1}{\sqrt{a}} \int_{-\infty}^{+\infty} s(z) \psi^{*}(z) d z
$$

family test function is derived from a single function $\psi(z)$ defined to as the analyzing wavelet according to [19]:

$$
\psi_{a, b}(z)=\psi\left(\frac{z-b}{a}\right)
$$

where $a \in R^{+*}$ is a scale parameter, $b \in R$ is the translation and $\psi^{*}$ is the complex conjugate of $\psi$. The analyzing function $\psi(z)$ is generally chosen to be well localized in space (or time) and wavenumber. Usually, $\psi(z)$ is only required to be of zero mean, but for the particular purpose of multiscale analysis $\psi(z)$ is also required to be orthogonal to some low order polynomials, up to the degree $n-1$, i.e., to have $n$ vanishing moments :

$$
\int_{-\infty}^{+\infty} z^{n} \psi(z) d z=0 \text { for } 0 \leq n \leq p-1
$$

According to equation (7), $p$ order moment of the wavelet coefficients at scale $a$ reproduce the scaling properties of the processes. Thus, while filtering out the trends, the wavelet transform reveals the local characteristics of a signal, and more precisely its singularities.

It can be shown that the wavelet transform can reveal the local characteristics of $s$ at a point $z_{0}$. More precisely, we have the following power-law relation [13],[20]:

$$
\left|C_{s}\left(a, z_{0}\right)\right| \approx a^{h(z o)}, \text { when } a \rightarrow 0^{+}
$$

where $h$ is the Hölder exponent (or singularity strength). The Hölder exponent can be understood as a global indicator of the local differentiability of a function $\mathrm{s}$.

The scaling parameter (the so-called Hurst exponent) estimated when analysing process by using Fourier Transform [13] is a global measure of self-affine process, while the singularity strength $h$ can be considered as a local version (i.e. it describes 'local similarities') of the Hurst exponent. In the case of monofractal signals, which are characterized by the same singularity strength everywhere $(h(z)=$ constant $)$, the Hurst exponent equals $h$. Depending on the value of $h$, the input signal could be long-range correlated $(h>0.5)$, uncorrelated $(h=0.5)$ or anticorrelated $(h<0.5)$.

\section{B. THE FONCTION OF PARTITION OF THE SPECTRUEM OF EXPONENTS}

The positioning of maxima is carried out using the calculation of the first and the second derivative of the modulus of the wavelets coefficients $|\mathrm{C}(\mathrm{a}, \mathrm{b})|$.

i.e.: $|\mathrm{C}(\mathrm{a}, \mathrm{b})|$ able a local maximum at the point $\mathrm{b}_{\mathrm{i}}$ If and only if :

$$
\left.\frac{\partial|C(a, b)|}{\partial b}\right|_{b=b i}=0 \quad \text { and }\left.\quad \frac{\partial^{2}|C(a, b)|}{\partial b^{2}}\right|_{b=b i} \prec 0
$$

The function of partition is the sum of the modulus of the wavelet coefficients on the local maxima, with a power of order q.

For low dilations the function of partition is dependent to 
the spectrum of exponent by : $Z(q, a) \approx a^{\tau(q)}$

By consequences the spectrum of exponents is obtained by a simple linear fit of $\log (\mathrm{Z}(\mathrm{q}, \mathrm{a}))$ versus $\log (\mathrm{a})$.

\section{THE SPECTRUM OF EXPOENTS}

Estimation of spectrum of exponent is based on the direct Legendre transform of the spectrum of exponents [14].

$$
D(h)=\min _{q}(q h-\tau(q))
$$

In our algorithm we use the functions defined in [14], based on Boltzmann's weights. These functions are defined as:

$$
\left\{\begin{array}{l}
\prec h \succ(q, a)=\sum_{\left(b_{i}, a\right)} \hat{T}_{\psi}[S]\left(q, b_{i}, a\right) \operatorname{Ln}\left|T_{\psi}[S]\left(b_{i}, a\right)\right| \\
D(q, a)=\sum_{\left(b_{i}, a\right)} \hat{T}_{\psi}[S]\left(q, b_{i}, a\right) \operatorname{Ln}\left|T_{\psi}[S]\left(q, b_{i}, a\right)\right|
\end{array}\right.
$$

where $\hat{T}$ is the Boltzmann weight defined by :

$$
\begin{aligned}
& \hat{T}_{\psi}[S](q, b, a)=\left|T_{\psi}[S]\left(b_{i}, a\right)\right|^{q} / Z(q, a) \\
& h(q)=\lim _{a \mapsto 0^{+}} \frac{1}{\operatorname{Ln}(a)} h(q, a) \\
& D(q)=\lim _{a \mapsto 0^{+}} \frac{1}{\operatorname{Ln}(a)} D(q, a)
\end{aligned}
$$

The spectrum of exponents is obtained by the graphical representation of $D(q)$ versus $h(q)$ for different values of $\mathrm{q}[14] . T_{\psi}[S]$ is the continuous wavelet transform of the $\mathrm{S}(\mathrm{t})$ signal.

\section{Optimization OF THE Processing PARAMETERS}

Theoretically the spectrum of exponents of an $\mathrm{fBm}$ signal is a segment of a straight line written as [14]:

$$
\tau(q)=q H-1
$$

$\mathrm{H}$ : is the Hurst exponent.

This stage consists to optimizing the WTMM processing parameters, by checking the linearity of the spectrum of exponents. Parameters to be optimized are:

1) The maximum value of the scale factor $q_{\max }$ where the calculation of the function of partition is carried out on the interval $\left[-\mathrm{q}_{\max },+\mathrm{q}_{\max }\right]$.

2) Parameters of the analyzing wavelet, which is the complex Morlet wavelet. It is written as:

$$
\psi(t)=\exp (i \omega t) \exp (-t \quad-\sqrt{ } \quad-\Omega \quad l i t \quad-t
$$

Several experiments on $\mathrm{fBm}$ realizations showed that the optimal value of $\Omega$ for a better estimation of the Hurst exponent is equal to 4.8 .

\section{A. DISCUSSION OF THE CHOICE OF THE COMPLEX MORLET WAVELET}

Two characteristics are important for any family of wavelets either continuous or discrete. These properties refer to the number of vanishing moments possessed by the wavelet and the regularity of the wavelet. The first propriety is defined as:

A Wavelet is said to have $M$ vanishing moments, if and only if for all positive integer $\mathrm{m}<\mathrm{M}$, it satisfies :

$$
\int_{-\infty}^{+\infty} z^{m} \psi(z) d z=0
$$

This definition shows to which order of polynomial the wavelet are orthogonal. It was showed [13] that the admissibility condition requires at least one vanishing moment, a notion being reflected in the fact that the wavelet contains at least one zero crossing. The number of zero crossing increases for increasing number of vanishing moments $\mathrm{M}$. The number of vanishing moments is directly linked to the regularity of the Fourier transform of the wavelet at the origin.

The Second important property of the wavelet concerns the differentiability and this corresponds to the decay rate of the Fourier coefficients as the frequency is being increased, i.e. $k \rightarrow \infty$.

It was demonstrated that an erroneous choice of the analyzing wavelet limits the detectable range of singularities. For that we will use The Morlet Wavelet because:

1 -It is characterized by infinity of vanishing moments:

We can demonstrate easily that for any $m \geq 0$

$$
\int_{-\infty}^{+\infty} z^{m} \psi(z) d z \approx 0
$$

2-It was demonstrated the existence of an intricate relationship between the smoothness of a function and its Fourier transform [13]. Given this relationship it possible to come up with estimates for the degree of regularity by taken the superior bound of all exponents $\alpha$ that satisfies the following condition:

$$
\int_{-\infty}^{+\infty}\left|\psi(k)(1+|k|)^{\alpha}\right| d k<\infty
$$

In this way we can demonstrate also that The complex Morlet wavelet check this condition.

\section{B. PROCESSING OF AN FBM SIGNAL WITH 1024 SAMPLES}

The first signal to be processed is an fBm model with a Hurst exponent $\mathrm{H}=0.60$ and 1024 samples. The realization of this last is represented in the Fig. (1.a) and the modulus of the wavelet coefficients is represented in Fig. $1 \mathrm{~b}$.

The skeleton of the wavelet coefficients is represented in Fig. (2.a). Physically each position in the skeleton can be a point of contact between two facies. The function of partition is represented in the half plan log-log in Fig.2.b. This last is calculated in the interval $[-2,+2]$ with a step of 0.125 .

The spectrum of exponents and the spectrum of singularities are represented in Fig.3a and Fig.3b. The solid line is the straight-line fit. One can remark that the spectrum of exponents is nonlinear and the spectrum of singularities is not concentrated in one point, which proves the erroneous choice value of $\mathrm{q}_{\max }$.

The spectrums of exponents calculated for $\mathrm{q}_{\max }=1.5, \mathrm{q}_{\max }$ $=1$ and $\mathrm{q}_{\max }=0.5$ are represented in Fig. 4 .a, Fig. $4 \mathrm{~b}$ and Fig. $4 \mathrm{c}$. Table I summarizes the values of $\mathrm{H}$ estimated for each value of $\mathrm{q}_{\text {max.. }}$ One can remark that the spectrum of exponents and the spectrum of singularities calculated for $\mathrm{q}_{\max }=0.50$ show that this value is optimal for the following points:

-Linearity of the spectrum of exponents which indicate the homogeneity of this texture 


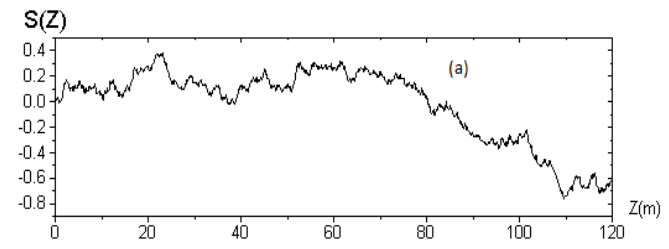

-As an estimator of the coefficient of roughness.

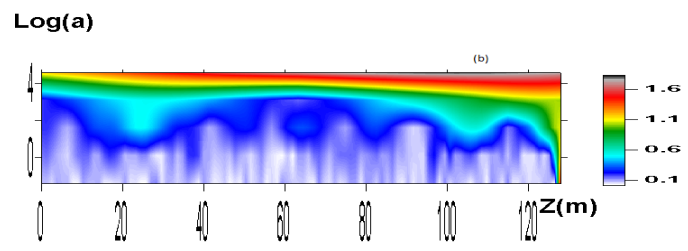

Fig..1 (a): fBm realization with 1024 samples and $\mathrm{H}=0.60$

(b) : Modulus of the wavelet coefficients in the plan half plan depth - log-dilations

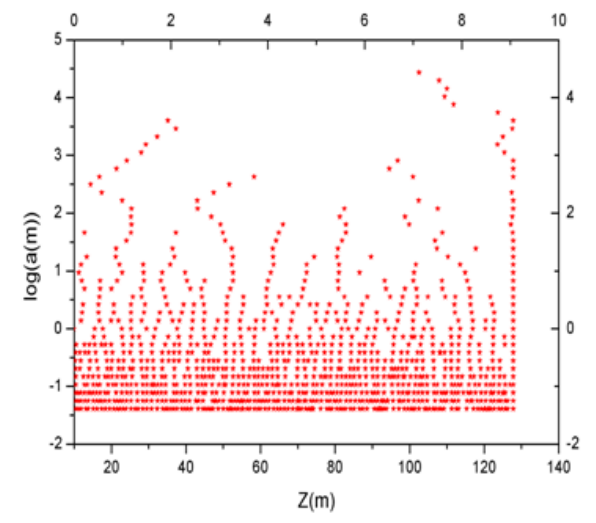

(a)

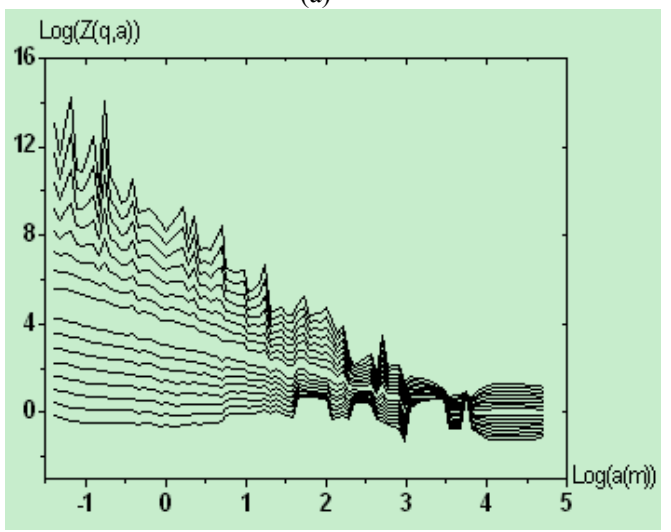

(b)

Fig. 2 (a) Skeleton of the module of the WT (b) Log the function of partition versus log-dilations.

Our objective is to seek very fine textures for that we have to concentrate our studies on short range series. We have analyzed sets of signals with 128, 64 and 32 samples. First we built several fbm realizations with 128 samples which different by their coefficients of roughness, it is varied from 0 to 1 . A detailed study showed that optimal values of $\mathrm{q}_{\max }$ are related to the Hurst exponent. Table II summarizes all obtained results.

Table I summarizes the values of $\mathrm{H}$ estimated for each value of $\mathrm{q}_{\text {max.. }}$ One can remark that the spectrum of exponents and the spectrum of singularities calculated for $\mathrm{q}_{\max }=0.50$ show that this value is optimal for the following points: -For the linearity of the spectrum of exponents which indicate the homogeneity of this texture
-As an estimator of the roughness coefficient.

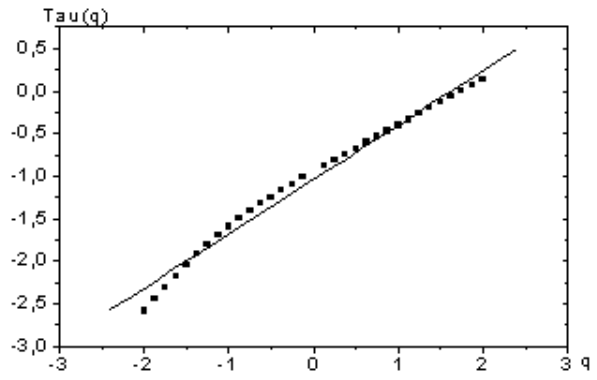

(a)

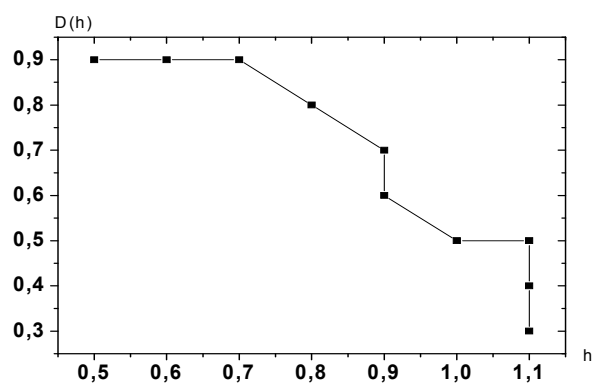

(a)

Fig.3 (a) Spectrum of exponents for $q \max =2$. (b) Spectrum of singularities

TABLE I: COEFFICIENTS OF HURST ESTIMATED VERSUS q $\max _{\text {. }}$

\begin{tabular}{|c|c|c|}
\hline $\mathrm{q}_{\max }$ & H injected & H calculated \\
\hline 2.0 & 0.60 & $0.685 \pm 0.010$ \\
\hline 1.5 & 0.60 & $0.664 \pm 0.005$ \\
\hline 1.0 & 0.60 & $0.685 \pm 0.006$ \\
\hline 0.5 & 0.60 & $0.636 \pm 0.03$ \\
\hline
\end{tabular}

TABLE.II OPTIMAL PARAMETERS ACCORDING TO ROUGHNESS

\begin{tabular}{|l|l|}
\hline $\mathrm{H}$ & $\mathrm{q}_{\max }$ \\
\hline $0 \prec H \leq 0.20$ & 0.125 \\
\hline $0.20 \prec H \leq 0.40$ & 0.25 \\
\hline$H \geq 0.40$ & 0.50 \\
\hline
\end{tabular}

To enhance the estimation, we have calculated the Hurst exponent by the DFA, obtained results showed that this estimator gave better estimation of the Hurst exponent compared to the WTMM.

Same work was made for signals with 64 and 32 samples; we have obtained the following results:

- WTMM analysis showed that the two types of signals able the same optimal parameters as those obtained for the signals with 128 samples.

- DFA Estimator gives better results than the WTMM.

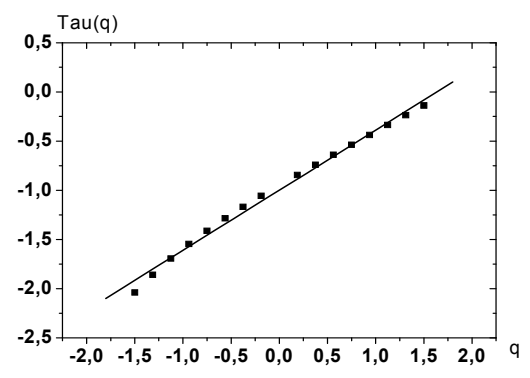

(a) 


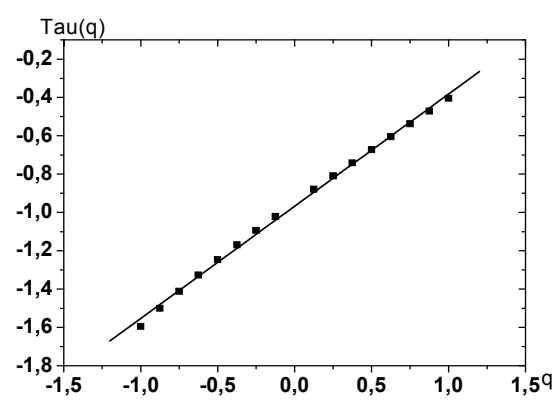

(b)

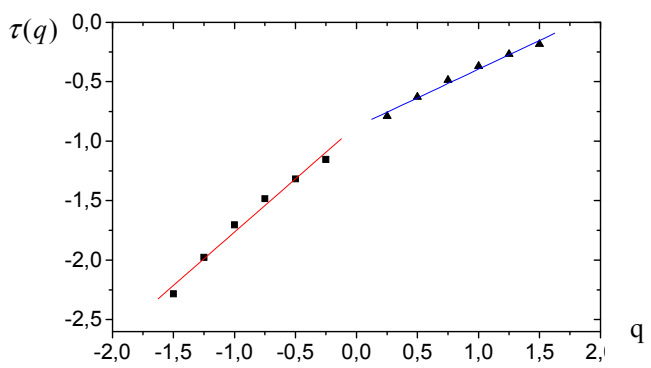

(c)

Fig. 4 Spectrum of exponents calculated for: (a) $\mathrm{q}_{\max }=1.5$ (b) $\mathrm{q}_{\max }=1$. (c) $\mathrm{q}_{\max }=0.50$

\section{ANALysis OF PhysicAl RESPONSE OF SEVERAL TEXTURES}

The theory developed by Arneodo shows [14]:

$\mathrm{S}(\mathrm{t})$ is a signal constitutes of a whole of $\mathrm{fBm}$ process of Hurst exponents $H_{1}, H_{2}, H_{3}, \ldots, H_{n}$, the spectrum of exponents $\tau(q)$ calculated by the WTMM formalism is depends only on the maximal and minimal Hurst exponents. The spectrum of exponents consists of two segments of straight lines of equations (15):

$$
\tau(q)=\left\{\begin{array}{l}
q H_{\max }-1 \ldots \ldots . i f(q \prec 0) \\
q H_{\min }-1 \ldots \ldots . i f(q \succ 0)
\end{array}\right.
$$

\section{A. Application on synthetic data}

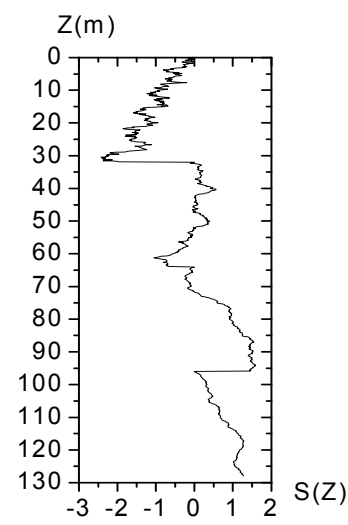

Fig. 5 Svnthetic model with 04 roughness

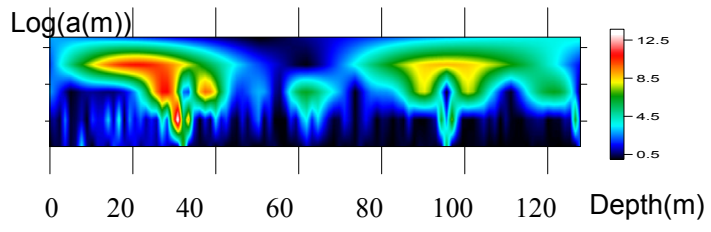

(a)

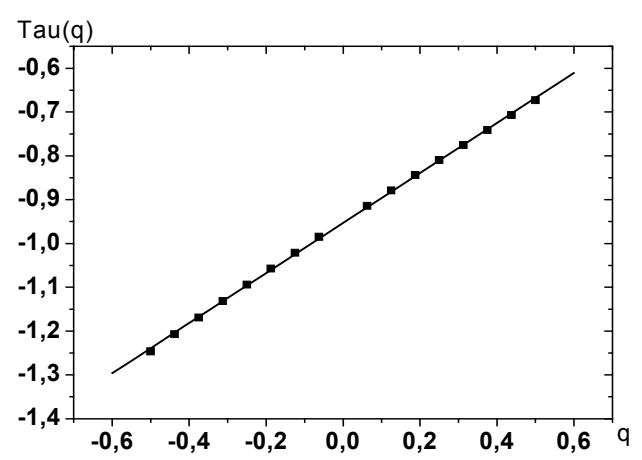

(b)

Fig. 6.WTMM analysis of the signal of the fig.5

(a) Modulus of the wavelet coefficients

(b) Spectrum of exponents

One can remark that the WTMM is sensitive only to the following two textures:

-The first, which has a maximum coefficient of roughness.

-The second characterized by a minimal roughness.

In order to check our source codes developed in $\mathrm{C}$ language we have generated a model made up of $04 \mathrm{fBm}$ realizations, with the following Hurst exponents: 0.40, 0.60, 0.70 and 0.80 , each signal has 64 samples. The realization of this model is represented in the Fig.(5). We applied a WTMM analysis to this signal, the modulus of the wavelets coefficients and the spectrum of exponents are represented in Fig.6.b and Fig.6.a.

\section{THE Processing AlgorithM}

Our algorithm of segmentation is based on the sensitivity of the WTMM of more than one homogeneous texture; this last phenomenon is expressed by two segments of straight lines in the spectrum of exponents. Estimation of roughness coefficient of each texture is enhanced by DFA. The input of the program of segmentation is composed of two variables:

a) Threshold of decision of homogeneity of textures, which it is equal to the difference between the slopes of the two segments of straight lines calculated for $\mathrm{q}<0$ and $\mathrm{q}>0$. We indicate by $\Delta H$ this variable in the flow chart and $\Delta \mathrm{z}$ is the sampling interval.

b) Minimal size where texture is considered homogeneous, we indicate by $\mathrm{W}$ this length.

The flow chart of the algorithm of segmentation is represented in Fig. (7).

\section{APPLICATION ON SYNTHETIC DATA}

We applied this algorithm at a whole of fBm realizations, with different Hurst exponents in order to model geological diversity. The modeled well-log consists of $02 \mathrm{fBm}$ signals, each signal has 64 samples. Their Hurst exponents are equals 
to 0.4 and 0.8 .

Fig. 8 represents the synthetic model with the obtained segmentation, in the half-plane depth - coefficient of roughness. The thickness of each column represents the thickness of the layer cut out and its height represents the value of the Hurst exponent estimated either by WTMM (black) or by DFA (red). The signal in green is the normalized $\mathrm{fBm}$ process. All details of segmentation are summarized in table 2 .

\section{APPLICATION ON REAL DATA}

The proposed idea has been applied at a borehole HFR located in the Berkine basin, it is a vast Paleozoic formation located in the South East of Algeria. It represents a very important hydrocarbon field.

\section{A. GEOLOGICAL CONTEXT OF THE BERKINE BASIN}

The Berkine basin is a vast circular Palaeozoic depression, where the basement is situated at more than $7000 \mathrm{~m}$ in depth. Hercynian erosion slightly affected this depression because only Carboniferous and the Devonian are affected at their borders. The Mesozoic overburden varied from $2000 \mathrm{~m}$ in Southeast to $3200 \mathrm{~m}$ in the Northeast. This depression is an intracratonic basin which has preserved a sedimentary fill out of more than $6000 \mathrm{~m}$. It is characterized by a complete section of Palaeozoic formations spanning from the Cambrian to the Upper Carboniferous. The Mesozoic to Cenozoic buried very important volume sedimentary material contained in this basin presents an opportunity for hydrocarbons accumulations. The Triassic province is the geological target of this study. It is mainly composed by the Clay and Sandstone deposits.

Its thickness can reach up to $230 \mathrm{~m}$. The Sandstone deposits constitute very important hydrocarbon reservoirs. The SIF FATIMA area where the borehole data are collected is restricted in the the labelled $402 \mathrm{~b}$ block.

It is located in the central part of the Berkine basin (See Fig.9a). The hydrocarbon field is situated in the eastern erg of the basin characterised also by high amplitude topography.

The studied area contains many drillings. However this paper will be focused on the HFR borehole. The main reservoir, the Lower Triassic Clay Sandstone labelled TAGI, is represented by fluvial and eolian deposits. The TAGI reservoir is characterised by three main levels: Upper, middle, and lower. Each level is subdivided into a total of nine subunits according to SONATRACH nomenclature [21].The lower TAGI is often of a very small thickness. It is predominantly marked by clay facies, sometimes by sandstones and alternatively by the clay and sandstone intercalations, with poor petrophysical characteristics (Fig. 9b).

\section{B. AUTOMATIC SEGMENTATION}

We applied this technique of segmentation at the HFR borehole located in the Berkine basin; we have processed the Gamma ray well-log data. Fig. (10) shows this well-log; the step of sampling is equal to $0.125(\mathrm{~m})$. Segmentation given in the stratigraphic column is used as a priori information, by consequences we have cut out each interval of the stratigraphic column. The various intervals of this last one are detailed in tableIII. Obtained lithofacies models of the three intervals are schematized in Fig.11. The right track of each scheme represents the obtained segmentation, however the left one is the gamma ray based model of lithofacies. Conclusion

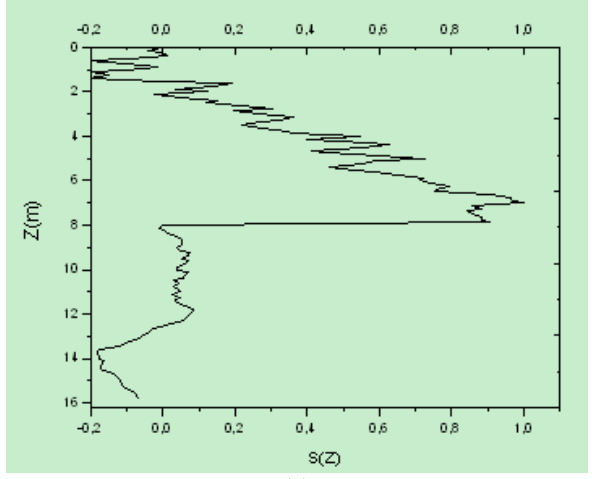

(a)

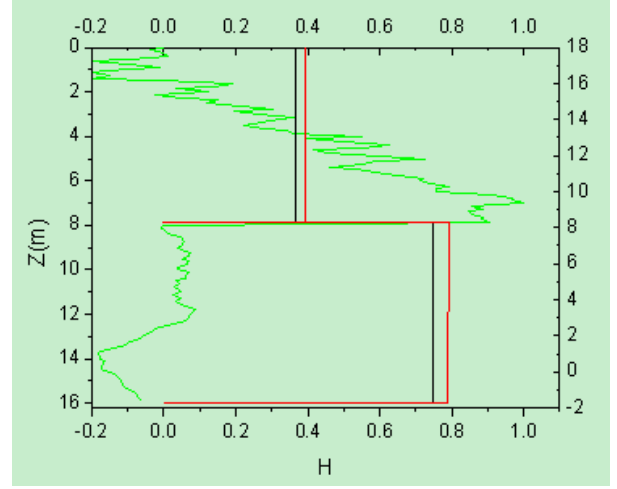

(b)

Fig. 8 Application of the algorithm of segmentation on a synthetic model.

(a) The synthetic model (b) Obtained segmentation

TABLE 2 OBTAINED SEGMENTATION OF THE SYNTHETIC MODEL WITH TWO ROUGHNESSES

\begin{tabular}{|c|c|c|c|c|c|}
\hline Layer & H injected & $\begin{array}{c}\text { Depth } \\
\text { injected }(\mathrm{m})\end{array}$ & Depth calculated & $\begin{array}{c}\text { H calculated } \\
\text { (WTMM) }\end{array}$ & $\begin{array}{c}\text { H calculated } \\
\text { (DFA) }\end{array}$ \\
\hline 1 & 0.40 & 7.875 & 7.875 & 0.363 & 0.392 \\
\hline 2 & 0.80 & 7.875 & 7.875 & 0.749 & 0.794 \\
\hline
\end{tabular}

We planned an automatic algorithm of segmentation, based on the sensitivity of the WTMM; the spectrum of exponents is an indicator of homogeneities of textures. We construct the lithofacies of the borehole HFR located in Algeria Sahara. Obtained lithofacies are compared with lithology units based of the gamma ray log (See the first track of figures $11 \mathrm{a}, 11 \mathrm{~b}$ and $11 \mathrm{c}$ ); this last provides information about shale percentage on the rock. Obtained results exhibit a big correlation between the two models.

The aim of this study is to realize a more consistent lithologic interpretation of logs optimising the use of the multifractal analysis resisted by the continuous wavelet transform. A technique of lithofacies segmentation based on the wavelet transform modulus maxima lines WTMM combined with the detrended fluctuation analysis DFA is developed and successfully applied the well log data of HFR borehole located in Berkine Basin in order to classify lithofacies. It is important to outline, that the comparison of data sets with classification derived from the Gamma ray log is legitimate because the studied interval is a limited part of the TAGI. Our results suggest an enhanced facies segmentation which leads an accurate interpretation process to update the reservoir architecture.

By implementing our method, we have demonstrated that 
it is possible to provide an accurate geological interpretation within a short time in order to take immediate drilling and completion decisions, but also, in a longer-term purpose, to update the reservoir model. Since of its computational efficiency, it is proposed that the present methods can be further exploited for analysing large number of borehole data in other areas of interest.

\section{TABLE 3 INTERVALS OF THE STRATIGRAPHIC COLUMN}

\begin{tabular}{c|c|c}
\hline Interval & $\begin{array}{c}\text { Minimal } \\
\text { depth }(\mathrm{m})\end{array}$ & $\begin{array}{c}\text { Maximal } \\
\text { depth(m) }\end{array}$ \\
\hline $\mathrm{C} 1$ & 2985 & 3258 \\
\hline $\mathrm{C} 2$ & 3258 & 3327.5 \\
\hline $\mathrm{C} 3$ & 3327.5 & 3350 \\
\hline
\end{tabular}

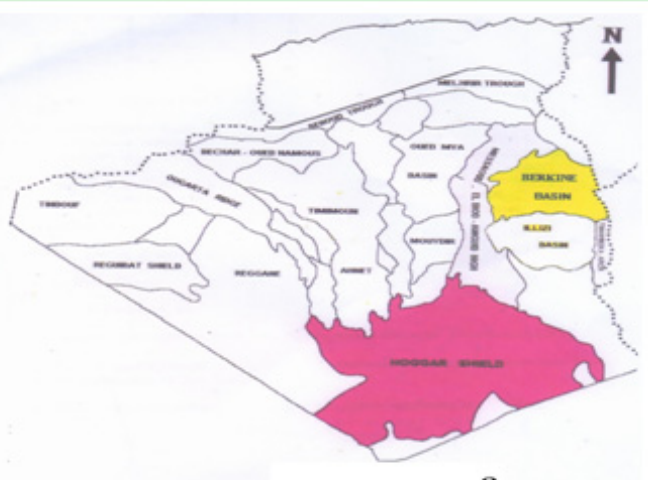

a

Fig. 9a. Geographic location of the Berkine Basin

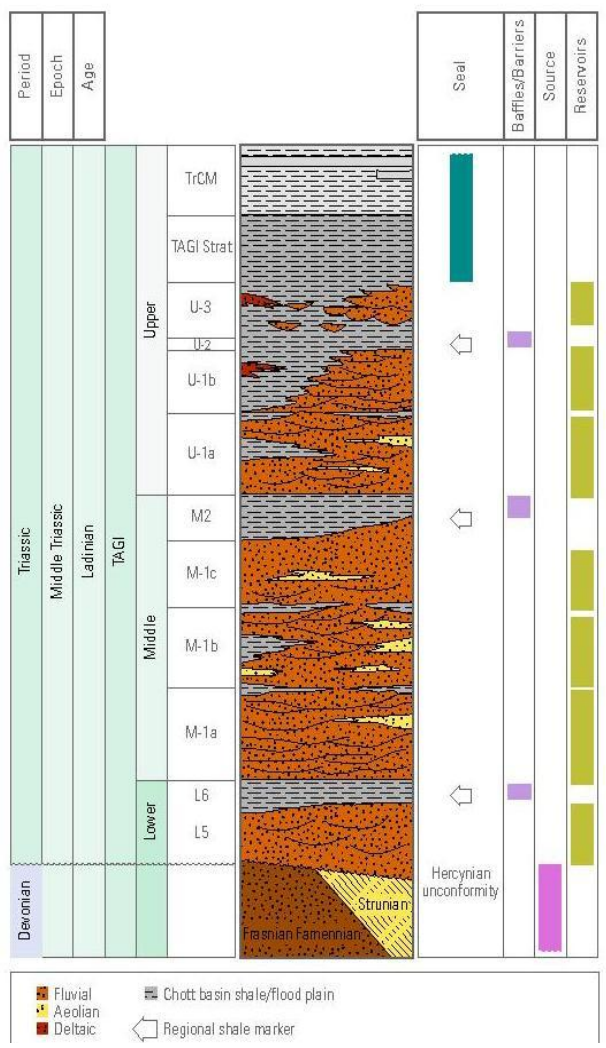

Fig. 9b.. Deposits age of the sets of the basins and the lithostratigraphy of the Triassic province.

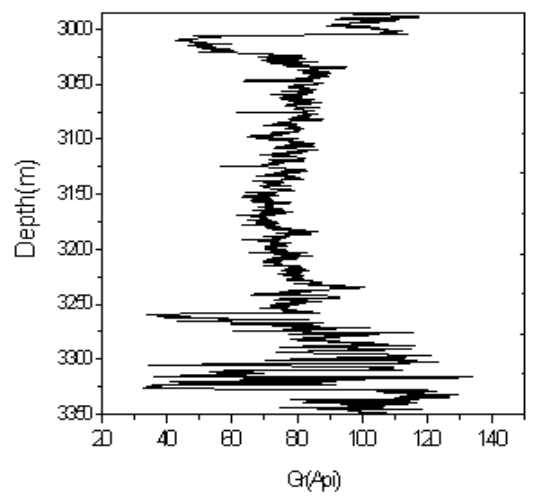

Fig.10. gamma ray well-log of the hfr borehole

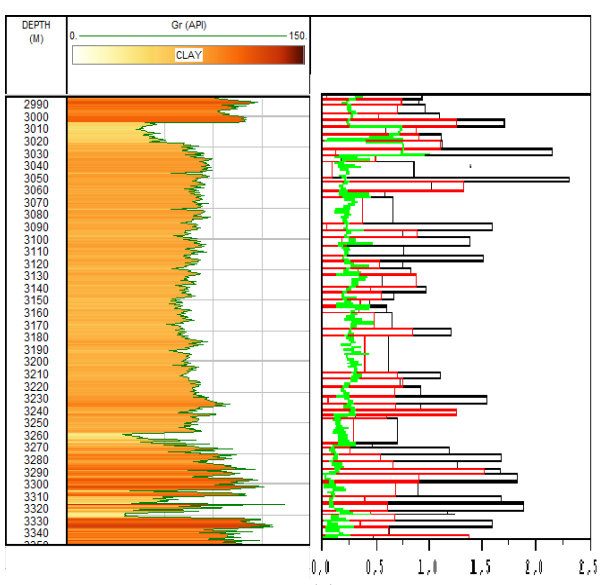

(a)

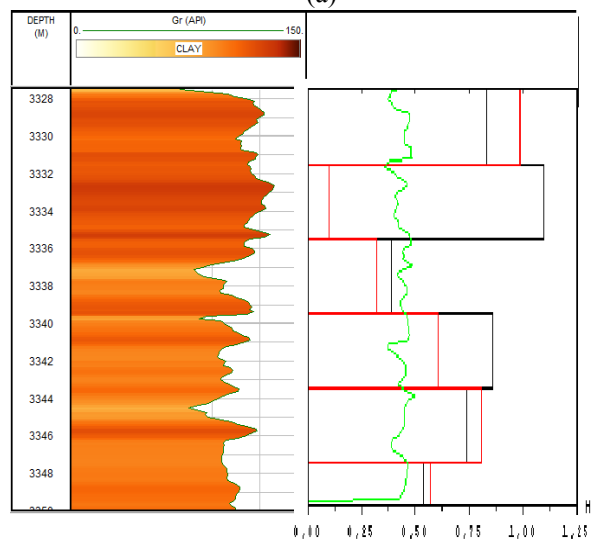

(b)

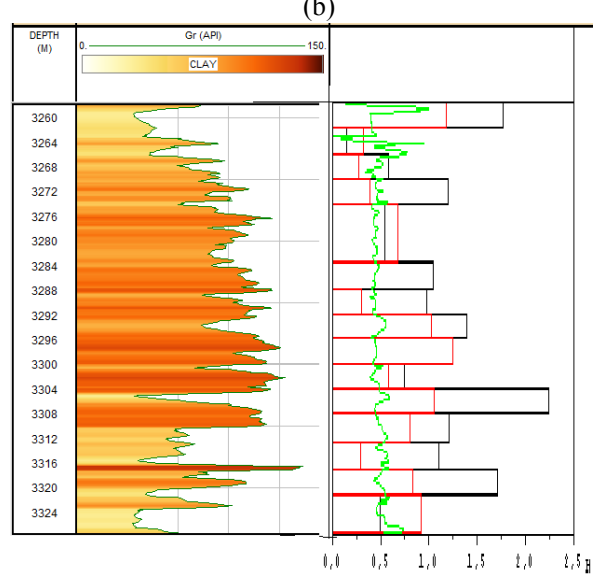

(c)

Fig.11. Lithofacies segmentation of the HFR borehole: Interval: (a) $\mathrm{C} 1$, (b) $\mathrm{C} 2$, (c) $\mathrm{C} 3$
Normalized signal 


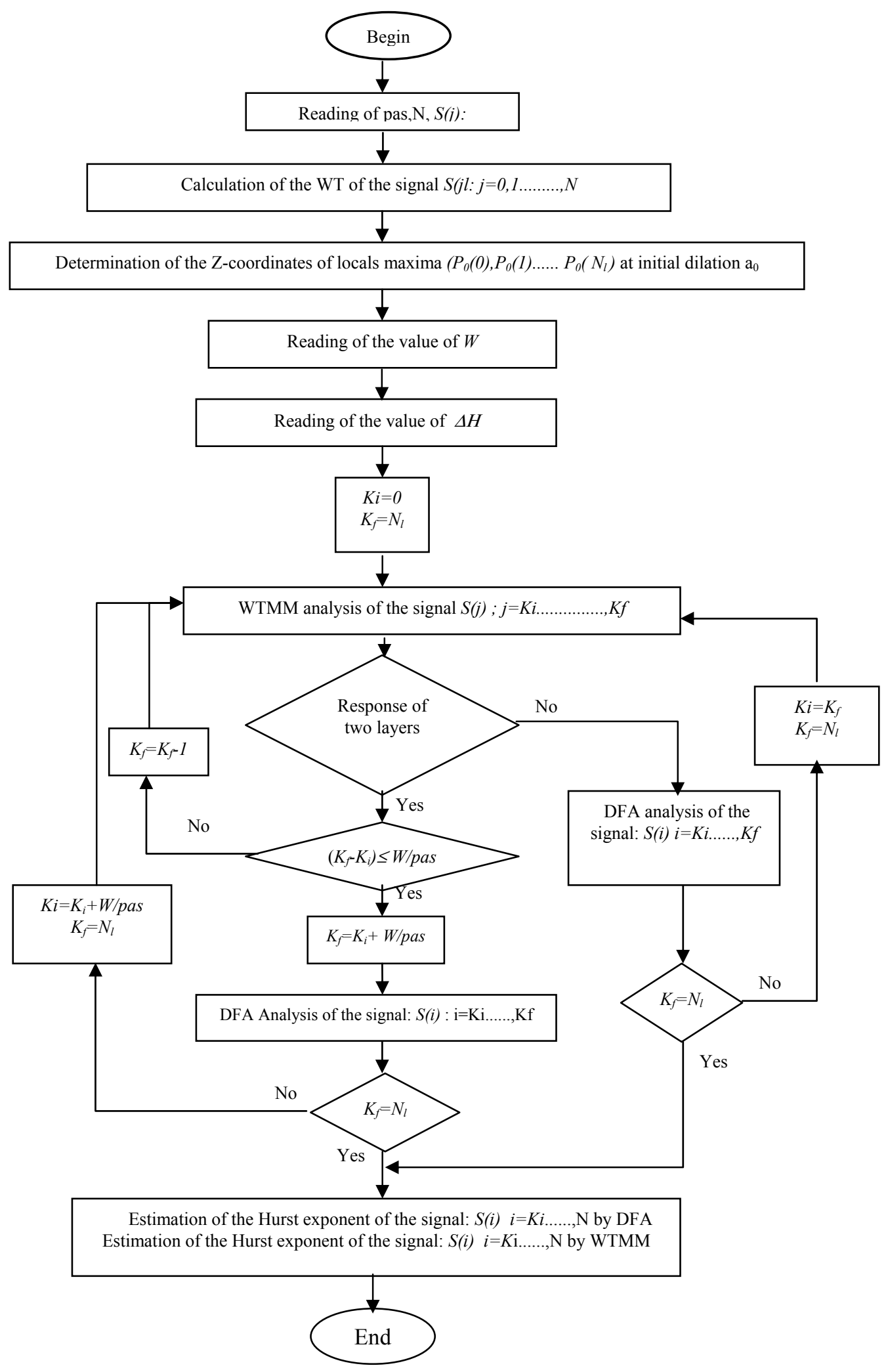

Fig. 7. Flow chart of automatic segmentation algorithm

\section{ACKNOWLEDGMENT}

The authors would like to thank Prof Alain Arneodo for his help, our thanks go to prof Lorenzo Crroco for his advising and suggestion on the article structuring.

\section{REFERENCES}

[1] [1] M.Pilkington, \& J.P.Tudoeschuck, "Naturaly smooth inversions with a priori information from well logs", Geophysics , vol.56,pp1811-1818,1991.

[2] [2] R.S.Wu , X.Zhengyu. , X.P.Li, "Heterogeneity spectrum and scale-anisotropy in the upper crust revealed by the German Continental Deep-Drilling (KTB) Holes", Geophys. Res. Lett, vol.21, pp911-914, 1994.

[3] [3] B.B. Mandelbrot, The fractal geometry of nature. Ed. W. H. Freeman, San Francisco, 1982. 
[4] G.Kneib, "The statistical nature of the upper continental cristalling crust derived from in situ seismic measurements", Geophys. J. Int., vol.122, pp594-616, 1982.

[5] C.J.Bean,. "On the cause of $1 / \mathrm{f}-$ power spectral scaling in borehole sonic logs, Geophys.Res. Lett., vol.23, pp. 3119-3122, $1982 .$.

[6] K.Holliger, "Upper crustal seismic velocity heterogeneity as derived from a variety of P-wave sonic log", Geophys. J. Int., vol.125, pp813-829, 1982 .

[7] D.L.Turcotte,.Fractal and Chaos in Geology and Geophysics, Cambridge University Press, Cambridge, 1997.

[8] Shiomi,K., Sato,H., Ohtake,M., 1997. Broad-band power-law spectra of well-log data in Japan, Geophys. J. Int. $130,57-64$.

[9] S.S.Dolan,. C.Bean, B.Riollet, "The broad-band fractal nature of heterogeneity in the upper crust from petrophysical logs", Geophys. J. Int., vol.132, pp489-507,1996.

[10] C-F.Li, .Rescaled-Range and power spectrum analysis on well-logging data, Geophys. J. Int. , vol.153 , pp201-212, 2003.

[11] S.Ouadfeul, Analyse de signaux gèophysiques par la technique des maxima du module de la transfromée en ondelettes MMTO, Thesis of Magister, USTHB , pp135,2006.

[12] S.Ouadfeul, Reservoir Characterization Using the Continuous Wavelet Transform (CWT) Combined with the Self Organizing Map (SOM), ECMOR, 2008.
[13] F.J.Herrmann, A scaling medium representation, a discussion on well-logs, fractals and waves ,Phd thesis Delft University of Technology, Delft, The Netherlands, pp.315, 1995.

[14] A.Arneodo, E.Bacry, Ondelettes ,multifractal et turbelance de l'ADN aux croissances cristalines, Diderot editeur arts et sciences ,Paris, 1995.

[15] C-K. Peng, S.V.Buldyrev, S.Havlin, M.Simons, , H.Stanley, .and A.L.Goldberger, , "Mosaic organization of DNA nucleotides", Phys.Rev. E, vol. 49, pp. 1685-1689, 1994.

[16] Li Dachang and Larry W .Lake,. A Moving Window Semivariance Estimator, Water Resources Research, 30, 5, pp. 1479-1489, 1994.

[17] A.Arneodo , G.Grasseau, and M.Holschneider, 1988. Wavelet transform of multifractals,Phys. Rev. Lett. 61:2281-2284, 1988.

[18] A.Grossman, and J-F.Morlet, J., Decomposition of functions into wavelets of constant shape, and related transforms , in :Streit, L., ed., mathematics and physics ,lectures on recents results , World Scientific Publishing, Singaporemm 1985

[19] B.Torrésani, Analyse continue par ondelettes, Inter Editions / CNRS Edition, 1995.

[20] B.Audit, E.Bacry, J-F.Muzy. And A.Arneodo, "Wavelet-Based Estimators of Scaling Behavior“, IEEE ,vol.48, pp. 2938-2954, 2002.

[21] S.Zeroug, N.Bounoua, R.Lounissi, 2007. Algeria Well Evaluation Conferencem, 2007.

http://www.slb.com/content/services/resources/premium_content.asp ?mode $=$ slb\& 